\title{
Data-Based Modelling of Significant Wave Height in the Adriatic Sea
}

\section{Luka Mudronja, Petar Matić, Marko Katalinić}

The paper deals with sea wave modelling based on available data acquired from a satellite-calibrated numerical model. The idea is to use an artificial neural network, as a flexible tool capable of modelling nonlinear processes, for significant wave height (SWH) modelling at a single point in the Adriatic Sea. The focus of the paper was not to develop a new type of ANN, but rather to use it as a modelling tool and identify the most significant input variables for SWH modelling in the Adriatic Sea, among the available data. Linear and nonlinear regression models were also developed for purposes of comparison of neural network performances with those of traditional data modelling methods. A total of 22 years of data were used - 20 years of data with a $6 \mathrm{~h}$ sampling step time, i.e. 30684 data samples were used to calibrate the models, while 2 years of data, i.e. 2920 data samples were used to test the models' performances. Simulation results proved the ability of an artificial neural network to model SWH with high accuracy based on available data. Furthermore, the artificial neural network model proved to be more accurate than traditional statistical models, especially when multiple input variables were used.

\section{KEY WORDS}

$\sim$ Sea wave

$\sim$ Data-based modelling

$\sim$ Artificial neural network

$\sim$ Regression analysis

$\sim$ Significant wave height

University of Split, Faculty of Maritime Studies, Split, Croatia

e-mail: luka.mudronja@pfst.hr

This work is licensed under (cc) BY

\section{INTRODUCTION}

Sea wave modelling is challenging due to its complexity, since a variety of parameters influence the mathematical description of the sea surface. In wave modelling in the Adriatic Sea, Tonko Tabain defined the Tabain spectrum of waves in the Adriatic Sea (Tabain, 1997). Computer development enabled numerical modelling and the application of numerical tools such as SWAN (Ris et al., 1999) to play a significant role in the sea wave modelling process. However, the use of numerical models might be limited by their complexity, high computational requirements and the need for accurate bathymetric data (Peres et al., 2015).

The availability of measurements and significant data bases made data-based modelling a viable option. Regression analysis, briefly described in section 3.1 of the paper, is a common tool in modelling systems based on available data. However, it requires deciding on which variables should be included in the model, the form such variables should take and, most importantly, the assumption of the functional relationship between dependent (output) and independent (input) variables of the system. These issues could be avoided by using an alternative, more flexible data-based modelling tool, such as artificial neural networks.

Artificial neural networks (ANNs), briefly presented in section 3.2 of this paper, have the ability to approximate nonlinear functions, which makes them an interesting tool for nonlinear system modelling. In essence, ANNs represent an approach similar to regression analysis, a method using data collected from a process to calculate model parameters. While regression analysis requires the definition of a strict mathematical form of a model, a neural network has a more flexible structure allowing it to adapt to the data. Therefore, while expected to yield similar results in simple cases, neural networks are anticipated to perform better in more complex cases where multiple variables influence the output variable. 
ANNs have already been used in ocean engineering mostly to model and/or predict significant wave height, as in (Zamani et al., 2009; Peres et al., 2015), peak wave period and, recently, wave energy flux, as reported in (Haddadpour et al., 2014). Input variables for sea wave modelling are usually wind speed and wind direction, with the previous values of the output variable also used as inputs. In paper (Peres et al., 2015), ANN was used to model significant wave height based on wind speed data, and the model was improved by expanding the input data set with more wind input points in the wave generation area. These predictions were used to fill-in the gaps in the significant wave height data series for three points in the Mediterranean Sea. In paper (Zamani et al., 2009), Kalman-filtering is applied to the ANN output to improve the network's prediction ability.

This paper examines the ability to apply feed-forward artificial neural network (ANN), known as multi-layer perceptron $(\mathrm{MLP})$, to model significant wave height $(\mathrm{SWH})$ at a single point in the Adriatic Sea, while examining the influence of different input variables.

Available data are presented in section 4.1 of this paper. In section 4.2, the results of correlation analysis are presented to pre-examine the influence of potential input variables on the output variable. In section 4.3, model-based experiments, used to determine the set of optimum input variables, are presented. For every ANN model, an appropriate regression model was also formed, and the comparison was made with the results presented and analysed in section 5 . The conclusions, based on the comparison of neural and regression model performances with the actual values of the output variable, are presented in section 6 .

\section{SEA WAVE MODELLING}

Wave modelling has a significant role in fields such as renewable energy planning, coastal engineering, naval architecture and maritime transportation. Sea surface waves are simultaneously an important energy resource that can be exploited by utilizing wave energy conversion devices (WEC) to generate electricity, and a threat to offshore installations and seagoing vessels owing to extreme loads capable of causing sudden structural collapses or "normal operation" long-term stress which can lead to fatigue damage. Detailed metocean studies facilitate the development of improved and safer designs by optimizing efficiency and minimizing risk.

Several methods can be applied to wave modelling, ranging from empirical to most sophisticated third-generation numerical models. Some examples of wave modelling approaches are as follows:

- $\quad$ modelling by formulated wave spectra function, e.g. Tabain spectrum, JONSWAP spectrum;

- $\quad$ numerical modelling with specialized software, e.g. SWAN,
WAM, WAVEWATCH III (Ortiz-Royero and Mercado-Irizarry, 2008); - $\quad$ modelling with wide-application tools, e.g. artificial neural networks.

Modelling with wave spectra function and specialized software are both well-known procedures for performing calculations in maritime transportation and naval architecture tasks in the Adriatic Sea (Katalinic et al., 2015). The use of numerical models might be limited by their complexity, high computational requirements and the need for accurate bathymetric data (Peres et al., 2015). The main goal of the present paper is to verify the application of a wide-range application tool, such as neural network, in wave modelling in the Adriatic Sea by examining available input variables for the case study.

\section{MODELLING METHODS}

As explained in (Rawlings et al., 2001), modelling refers to the development of mathematical expressions describing the behavior of a variable of interest. This variable is called the dependent variable and is usually denoted by $y$. Other variables which are thought to provide information on the behavior of the dependent variable are incorporated into the model as predictors or explanatory variables. These variables are called independent variables, and can be denoted by $x$, with additional notation as needed to identify different independent variables. In addition to dependent and independent variables, all models likewise involve unknown constants, called parameters, which control the behavior of the model. Since the modelling method should provide a way to determine the values of these parameters, the models' response should fit the available data. The basic idea of this paper is to use an artificial neural network as a modelling method in a case study and compare it with a traditional databased modelling method, i.e. regression analysis.

\subsection{Regression Analysis}

The simplest linear model involves only one independent variable and states that the dependent variable changes at a constant rate as the value of the independent variable increases or decreases. Thus, the functional relationship between $y_{i}$ and $x_{i}$ is the equation of a straight line (Rawlings et al., 2001), described with (1) for the univariate case.

$y_{i}=a_{1} x_{i}+a_{0}$

The subscript $i$ indicates the particular observational unit, the $y_{i}$ and $x_{i}$ pair of observations. The $x_{i}$ stands for $\mathrm{n}$ observations of the independent variable, while $y_{i}$ denotes the observations of the dependent variable. Therefore, a simple linear model has two parameters, $a_{0}$ and $a_{1}$, to be estimated from the data. 
If there was no random error in $y_{i}$, any two data points could be used to obtain the values of the parameters. A random variation in a set of $y_{i}$ values causes each pair of observed data points to give different results. Therefore, a method is needed that will combine all the information from the data into a single solution considered to be the "best" by some criterion. The least squares estimation, explained in (Rawlings et al., 2001), is frequently used for this purpose.

Models sometimes explain the behavior of the dependent variable by $k$ independent variables. In this case, the linear model can be extended, as described in (2), for the multivariate case, and the problem is called multiple regression analysis. In this case, there are $k+1$ parameters to be estimated, and the common least squares estimate can also be applied.

$y_{i}=a_{0}+a_{1} \cdot x_{1 i}+a_{2} \cdot x_{2 i}+L+a_{k} \cdot x_{k i}$

More realistic and therefore more complex models use nonlinear relationships between the dependent variable and independent variables. The introduction of higher-degree polynomials, exponential and trigonometric functions increases the flexibility of the model. Generally, these models can be expressed with (3), where $f_{N}$ stands for a nonlinear function.

$y_{i}=f_{N}\left(a_{k+1}, x_{k i}\right)$

Regression analysis requires the determination of the functional form of the model, which could cause errors. However, these decisions could be avoided by using an alternative, more flexible data-based modelling tool such as artificial neural networks.

\subsection{Artificial Neural Networks}

ANNs represent a simplified mathematical model of the process occurring in the brain of living beings. ANN is an artificial structure consisting of a number of interconnected artificial neurons, resembling the biological neural network in terms of information processing and storage, as described in (Haykin, 1999). Based on neuron type and their mutual connections, different ANNs have been developed over the last 50 years. So far, ANNs have been successfully applied to a large number of different computational problems such as pattern recognition, classification, function approximation, modelling and prediction.

A static feed-forward ANN called Multi-Layer Perceptron (MLP) is probably the most commonly used network architecture in most applications, especially function approximation, i.e. modelling and prediction. As briefly described in (Matić et al., 2015), MLP with m inputs, $h$ hidden neurons and o output neurons, uses nonlinear sigmoidal activation functions in a hidden layer, which enables it to approximate nonlinear functions, i.e. model non-linear processes. Although the optimum number of layers was often the subject of research, two-layer structure was proven to be sufficient to approximate any practical function, given enough neurons in the hidden layer (Cybenko, 1989). Therefore, a two-layer MLP, known as universal approximator, was used in this research to form a neural model of significant wave height (SWH).

For a model structure to be defined, the number of inputs (Ni), the number of hidden neurons (Nh) and the number of output neurons (No) have to be determined. The determination of input variables is case-dependent, and is therefore discussed in more detail in sections 4 and 5 of this paper. The number of output neurones is determined by the number of output variables and depends on the modelling goal, i.e. the model's purpose. In this case, it is set to one neuron. Since the quality of the model is dependent upon the number of hidden neurons, it is inevitably a subject of research in the model development process.

Although defined by its structure, i.e. neuron number and type, ANN takes its final form only after the completion of the learning process. The procedure used to execute the learning process is called the learning (or training) algorithm. Its function is to modify network parameters in an orderly fashion to obtain the desired objective, i.e. minimize error between network output and the desired value. The basic algorithm developed for MLP network training is error backpropagation (EBP), which could be considered one of the most significant breakthroughs in the field of neural networks, as noted in (Matic et al., 2015). Although EBP algorithm is still widely used, many improvements have been made to the original algorithm to deal with the issue of its slow convergence. In this paper, a Levenberg-Marquardt (LM) algorithm, explained in (Hagan and Menhaj, 1994) and (Yu and Wilamowski, 2011), and Bayesian regularization (BR), explained in (Foresee and Hagan, 1997), were used to train the network. BR can be a useful tool for determining the sufficient number of hidden neurons, improving on the Levenberg-Marquardt (LM) algorithm, already proven to be the fastest and most appropriate algorithm for training networks, possibly containing hundreds of adjustable parameters (Beale et al., 2010). To determine the optimum number of hidden neurons, BR was used in addition to a set of experiments on training ANNs with varying numbers of hidden neurons.

Artificial neural networks (ANNs) have been explained in detail in (Haykin, 1999), or (Beale et al., 2010) which is more focused on the application of ANNs as a modelling tool. Explicit examples of application in sea wave modelling can be found in references stated in the introduction. The intent of this paper was neither to develop a new type of ANN, nor improve an existing one, but rather to explore the possibility of application of ANN in Adriatic Sea wave modelling, i.e. significant wave height (SWH). 


\section{CASE STUDY - ADRIATIC SEA WAVE MODELLING}

The available data were obtained from an environmental monitoring company, Fugro OCEANOR, to be used in the DATAS (Damaged Tanker in The Adriatic Sea) project funded by the Croatian Science Foundation. The data were collected by satellite mapping between January 1992 - January 2016, with the time step of 6 hours, at 40 points in the Adriatic Sea. The calculations in this paper pertain to data from a single point in the Adriatic Sea with the following coordinates:

- latitude $42 \mathrm{~N}$
- longitude $17 \mathrm{E}$

The chosen point is located on the busy merchant ship route between Otrant (SE entrance into the Adriatic Sea) and NE Adriatic ports (Rijeka, Venice, Koper). All data were divided into 12 classes of variables (Table 1, 1-12), the names, abbreviations and units of which are presented in Table 1. Additionally, the authors generated two more potential input variables from available data, namely wind time of duration (WTD) to account for past wind behaviour, and the wind quadrant (WQ) to simplify wind direction information (Table 1, 13-14).

Table 1.

Available data.

\begin{tabular}{llll} 
Class & Variable name & Abbreviation & Unit \\
\hline 01 & Significant wave height & SWH & $\mathrm{m}$ \\
\hline 02 & Mean wave direction & MDIR & ${ }^{\circ}$ (degrees) \\
\hline 03 & Peak period of 1d spectra & PP1D & $\mathrm{s}$ \\
\hline 04 & Mean wave period & MWP & $\mathrm{m}$ \\
\hline 05 & Significant height of wind waves & SHWW & ${ }^{\circ}$ (degrees) \\
\hline 06 & Mean direction of wind waves & MDIRW & $\mathrm{s}$ \\
\hline 07 & Mean period of wind waves & MPWW & $\mathrm{m}$ \\
\hline 08 & Sign. height of primary swell & SHPS & ${ }^{\circ}$ (degrees) \\
\hline 09 & Mean direction of primary swell & MDIRS & $\mathrm{s}$ \\
\hline 10 & Mean period of primary swell & MPPS & $\mathrm{m} / \mathrm{s}$ \\
\hline 11 & Wind speed at 10 $\mathrm{m}$ height & WSP & ${ }^{\circ}$ (degrees) \\
\hline 12 & Wind direction at 10 $\mathrm{m}$ height & WDIR & $\mathrm{h}$ \\
\hline 13 & Wind time duration & WTD & {$[1-8]$} \\
\hline
\end{tabular}

\subsection{Data Analysis}

Data analysis is performed to examine the relations between potential input and output variables. Although physical processes are mostly non-linear, linear measures, such as correlation analysis, are often used due to their simplicity. Non-linear methods, such as Mutual Information (Zamani et al., 2009), can also be used. However, these results are never taken unequivocally, i.e. the results of data analysis are considered to be merely an assumption, and should be verified by means of a model-based experiment. In this case, the correlation analysis was performed to examine the influence of potential input variables, thus providing an insight into the interdependence of input and output variables. Correlation analysis results presented in Table 2 are used to facilitate the selection of input variables.

Table 2.

Correlation analysis for potential input variables for the chosen point in the Adriatic Sea.

\begin{tabular}{llllll} 
CC & WSP & WDIR & WQ & WTD & SWH $_{\mathbf{t}-1}$ \\
\hline SWH & 0.839392 & -0.09057 & -0.05716 & 0.171479 & 0.902132
\end{tabular}


Results from Table 2 suggest that $\mathrm{SWH}_{\mathrm{t}}$ is strongly influenced by $\mathrm{WSP}_{\mathrm{t}}$ and its own value from the preceding calculation step, $\mathrm{SWH}_{\mathrm{t}-1}$. Therefore, $\mathrm{WSP}_{\mathrm{t}}$ and $\mathrm{SWH}_{\mathrm{t}-1}$ are selected as the best potential inputs for the SWH model. These assumptions are validated by model-based experiments.

\subsection{Model Formulation}

Data sets obtained from Fugro OCEANOR and the DATAS project were used in the modelling process. From the available 22 years of data - 20 years of data were used to calibrate and 2 years of data to test the models. Sample rate, i.e. time step, was 6 hours, yielding 30683 samples for calibration and 2920 samples for model performance testing. Optimum input variables for modelling the current value of significant wave height $\left(\mathrm{SWH}_{\mathrm{t}}\right)$ were identified by examining a set of potential input variables through a number of experiments. Since correlation analysis suggested that $\mathrm{WSP}_{\mathrm{t}}$ and $\mathrm{SWH}_{\mathrm{t}-1}$ were the best potential inputs for SWH modelling, experiments were performed as described in Table 3.

Table 3.

Experiment setup for optimum input variable identification.

\begin{tabular}{ll}
$\boldsymbol{i}$ & Formulation \\
\hline $\mathbf{1}$ & $S W H_{t}=f\left(W S P_{t}\right)$ \\
\hline $\mathbf{2}$ & $S W H_{t}=f\left(W S P_{t^{\prime}} S W H_{t-1}\right)$ \\
$\mathbf{3}$ & $S W H_{t}=f\left(S W H_{t-1}\right)$ \\
\hline
\end{tabular}

Two models were formed for each experiment $i \in[1,3]$, a neural network model $\left(N N M_{i}\right)$, and a regression model $\left(\mathrm{RM}_{\mathrm{i}}\right)$. The number of hidden layer neurons for the neural models was determined by experiments, where the number of neurons was increased from 10 to 50 with the step size of 10. The BR algorithm was also used to optimize the number of network parameters. Since training outcome is dependent upon the initial values of network parameters $(w)$, multi-start was applied to bring the solution closer to the global minimum. This means that each network structure with different initial values of $w$ parameters is trained $P$ times, with the highest-ranking network selected as the representative model. $P=10$ was used as the optimum number of consecutive network trainings based on a recommendation from (de Vos and Rientjes, 2005). In the regression analysis, for every $\mathrm{RM}_{\mathrm{i}}$, a number of polynomial functions were explored to obtain the optimum solution, found to be a simple quadratic function.

\subsection{Model Evaluation}

Graphical and numerical methods can be used to evaluate a model's performances. Graphical methods enable visual comparison of a model's response to actual values, offering a simple first impression of the overall quality of the model. Numerical methods measure the exact quantity of deviation of a models' response from the actual value by means of statistical measures of quality.

Numerical evaluation of data-based modeling in the field of hydrology was analyzed by LeGates and McCabe (1999), with the results applicable to this case study as well. The authors of (LeGates and McCabe, 1999) proposed a set of different measures to evaluate the quality of the model, and recommended that the set include at least one relative and one absolute measure. In the paper (Gupta et al., 1999) the application of Persistence Index (PI) is also recommended. Therefore, the quality of a model is established by means of the following measures, absolute: root mean squared error (RMSE), mean absolute error (MAE); and relative: Nash-Sutchllife coefficient of efficiency (NSC, or CE), percent bias (PBIAS), RMSE to standard deviation ratio (RSR) and persistency index (PI). RMSE, MAE, CE, PBIAS, RSR and PI measures are defined with expressions (4) to (9), in the corresponding order.

$$
\begin{aligned}
& R M S E=\sqrt{\frac{1}{S} \sum_{s=1}^{S}\left(d_{s}-y_{s}\right)^{2}} \\
& M A E=\frac{1}{S} \sum_{s=1}^{s}\left|d_{s}-y_{s}\right|
\end{aligned}
$$

$C E=1-\frac{\sum_{s=1}^{s}\left(d_{s}-y_{s}\right)^{2}}{\sum_{s=1}^{s}\left(d_{s}-\bar{d}\right)^{2}}$

PBIAS $=\frac{\sum_{s=1}^{s}\left(d_{s}-y_{s}\right)}{\sum_{s=1}^{s}\left(d_{s}\right)} \cdot 100 \%$ 
$R S R=\frac{R M S E}{S T D E V} \sqrt{\frac{\sum_{s=1}^{s}\left(d_{s}-y_{s}\right)^{2}}{\sum_{s=1}^{s}\left(d_{s}-\bar{d}\right)^{2}}}$

$P I=1-\frac{\sum_{s=1}^{N}\left(d_{s}-y_{s}\right)^{2}}{\sum_{s=1}^{N}\left(d_{s}-d_{s-1}\right)^{2}}$
RMSE, MAE and RSR values range from high $(+\infty)$ to 0 , with lower values indicating lower errors. $C E$ and $\mathrm{PI}$ values range from negative $(-\infty)$ to 1 , where high positive values indicate a better fit. PBIAS measures the average propensity of the model for the calculation of output values $(y)$ lower or higher than the measurements $(d)$. The values of the criteria range from 0 to $\pm 100 \%$, with lower values being indicative of better model performance. Positive values indicate a model's bias toward underestimation, while negative values indicate bias toward overestimation.

In (Gupta et al., 1999) a satisfactory model is defined as the one with positive values of the PI coefficient. In paper (Moriasi et al., 2007), model classification is performed based on the values of CE, RSR and PBIAS quality measures. The same classification is presented in Table 4 and used in this paper to evaluate a model's performance.

Table 4

Model classification based on CE, RSR and PBIAS values.

\begin{tabular}{llll} 
Model quality & CE & RSR & PBIAS \\
\hline Very good & $(0.75,1.00]$ & {$[0.00,0.50]$} & $< \pm 10 \%$ \\
\hline Good & $(0.65,0.75]$ & $(0.50,0.60]$ & {$[ \pm 10, \pm 15) \%$} \\
\hline Satisfactory & $(0.5,0.65]$ & $(0.6,0.70]$ & $\geq \pm 15, \pm 25) \%$ \\
Unsatisfactory & $\leq 0.5$ & $>0.7$ & $\geq \pm 25 \%$
\end{tabular}

\section{RESULTS AND DISCUSSION}

The results of the numerical evaluation of neural and regression models for experiments 1 to 3 are shown in Table 5 . Due to high CE values and relatively low values of RSR, RMSE and MAE measures, NNM 1 and $\mathrm{RM}_{1}$ can both be noted to fairly model the system, with only the $\mathrm{PI}$ criterion measuring low negative value, indicating unsatisfactory model performances. Numerical evaluation of the models from experiment 2 is indicative of excellent model behaviour due to low RMSE, MAE, RSR and PBIAS values, and CE and PI measures yielding values close to 1. The $\mathrm{NNM}_{2}$ model can be noted to score slightly higher than $\mathrm{RM}_{2}$ by all numerical measures used. Numerical evaluation of the models from experiment 3 indicates satisfactory model behaviour by all measures used, with only PBIAS evaluating a $\mathrm{NNM}_{3}$ better than $\mathrm{RM}_{3}$. The overall comparison of the models' performances from Table 5 indicates that $\mathrm{NNM}_{2}$ is the best model for representing $\mathrm{SWH}$ behaviour in the case study.

Table 5 .

Numerical evaluation of the performance of models from experiments 1 to 3 .

\begin{tabular}{lllllll} 
Model & \multicolumn{3}{c}{ Absolute } & \multicolumn{2}{c}{ Relative } \\
\cline { 2 - 8 } & RMSE & MAE & PI & CE & PBIAS & RSR \\
\hline NNM1 & 0.293 & 0.203 & -0.178 & 0.791 & 2.918 & 0.52 \\
\hline RM1 & 0.293 & 0.204 & -0.179 & 0.79 & 2.937 & 0.52 \\
\hline NNM2 & 0.126 & 0.083 & 0.784 & 0.962 & 0.147 & 0.2 \\
\hline RM2 & 0.136 & 0.091 & 0.745 & 0.955 & 0.232 & 0.216 \\
\hline NNM3 & 0.264 & 0.18 & 0.044 & 0.83 & 0.93 & 0.459 \\
\hline RM3 & 0.264 & 0.18 & 0.043 & 0.83 & 1.148 & 0.458
\end{tabular}


Models from experiments 1 and 3 tend to give best results if $\mathrm{h}=10$ neurons from the hidden layer are used. Models from experiment 2 tend to give best results if $h=20$ neurons from the hidden layer are used. Optimum regression models are defined with expressions (10) to (12) for experiments 1 to 3 , in the corresponding order, where y stands for $\mathrm{SWH}$ and $\mathrm{x}$ represents the WSP variable.

$y_{t}=0.0119 \cdot x_{t}^{2}+0.0126 \cdot x_{t}+0.392$

$y_{t}=-0.0288 \cdot y_{t-1}^{2}+0.6556 \cdot y_{t-1}+0.084 \cdot x_{t}^{2}$

$+0.0166 \cdot x_{t}+0.1197$

$y_{t}=-0.0255 \cdot y_{t-1}^{2}+0.9726 \cdot y_{t-1}+0.0546$

The ordinate in Figures 1 to 3 represents significant wave height measured in meters, while abscissa represents time with a 6-hour step. For clearer presentation of the graphical evaluation, responses in Figures 1 to 3 are magnified, showing only model behaviour for sample 2740 to 2820 .
Graphical evaluation of performances of models from experiment 1, presented in Figure 1, indicates consistent error in the range of low values of the SWH variable. Graphical evaluation also shows the model's tendency to predict sudden variable changes, causing numerical measures to have a negative impact on model's performances, although it is actually doing more than just modelling in some cases, it is predicting. This only underscores the importance of use of numerical and graphical evaluation in conjunction, to get a proper evaluation of models' performances.

Graphical evaluation of models' performances from experiment 2, presented in Figure 2, shows excellent correspondence of models' responses and actual data.

Graphical evaluation of models' performances from experiment 3, presented in Figure 3, suggests that lag effect is a problem preventing both models $\left(\mathrm{NNM}_{3}\right.$ and $\left.\mathrm{RM}_{3}\right)$ from modelling sudden changes of the $\mathrm{SWH}$.

\section{CONCLUSION}

Based on the results presented in section 5 it can be concluded that artificial neural networks can be successfully applied to significant wave height modelling in the Adriatic Sea. The models from experiment 1, using only WSP to model SWH, although being slightly under-rated by statistical measures of performance, have manifested an interesting ability to predict sudden changes of the output variable. This conclusion should be exploited in terms of SWH prediction in further research.

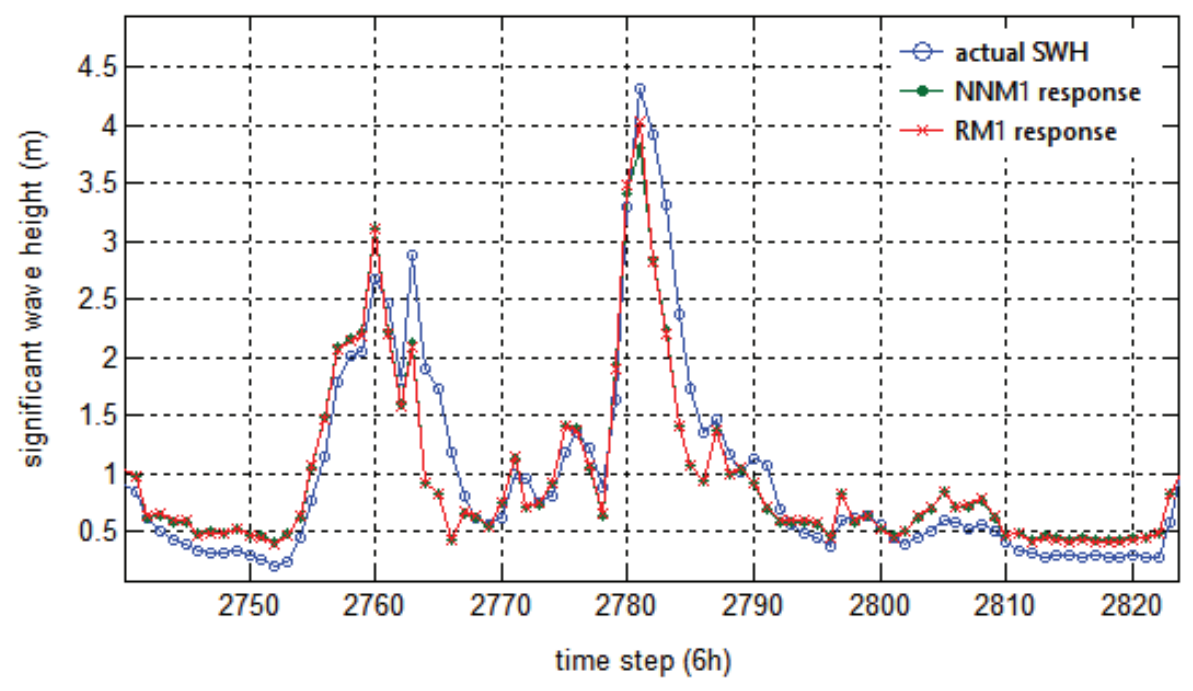

Figure 1.

Graphical comparison of model responses to actual SWH values from experiment 1. 


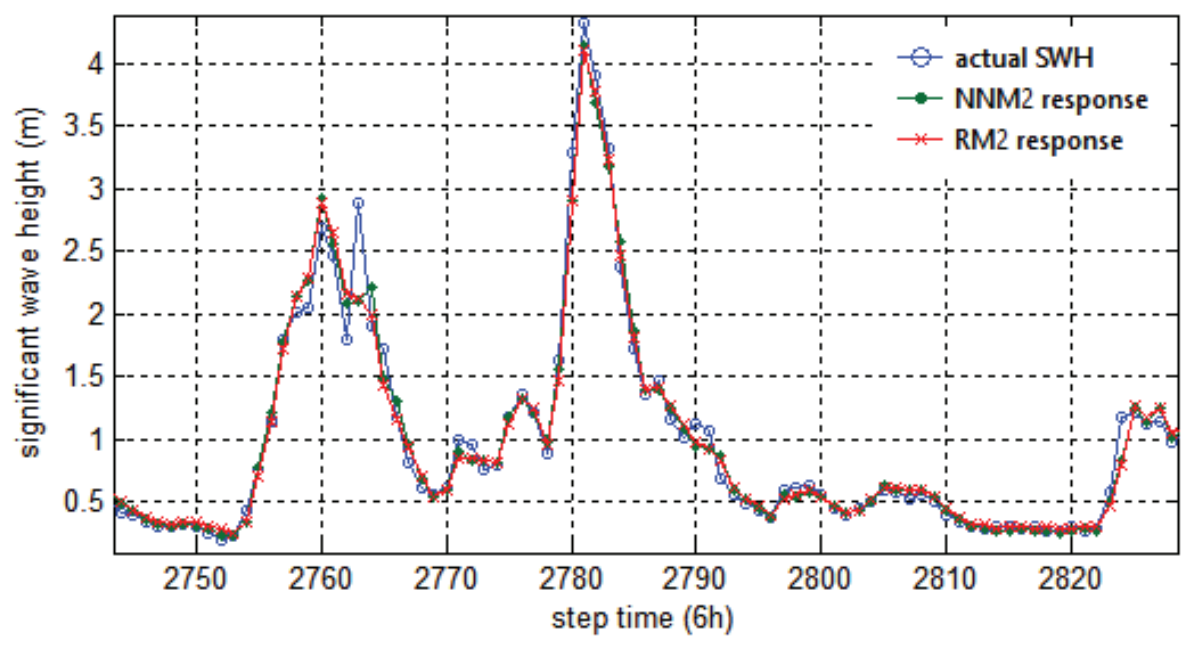

Figure 2.

Graphical comparison of models' responses to actual SWH values from experiment 2.

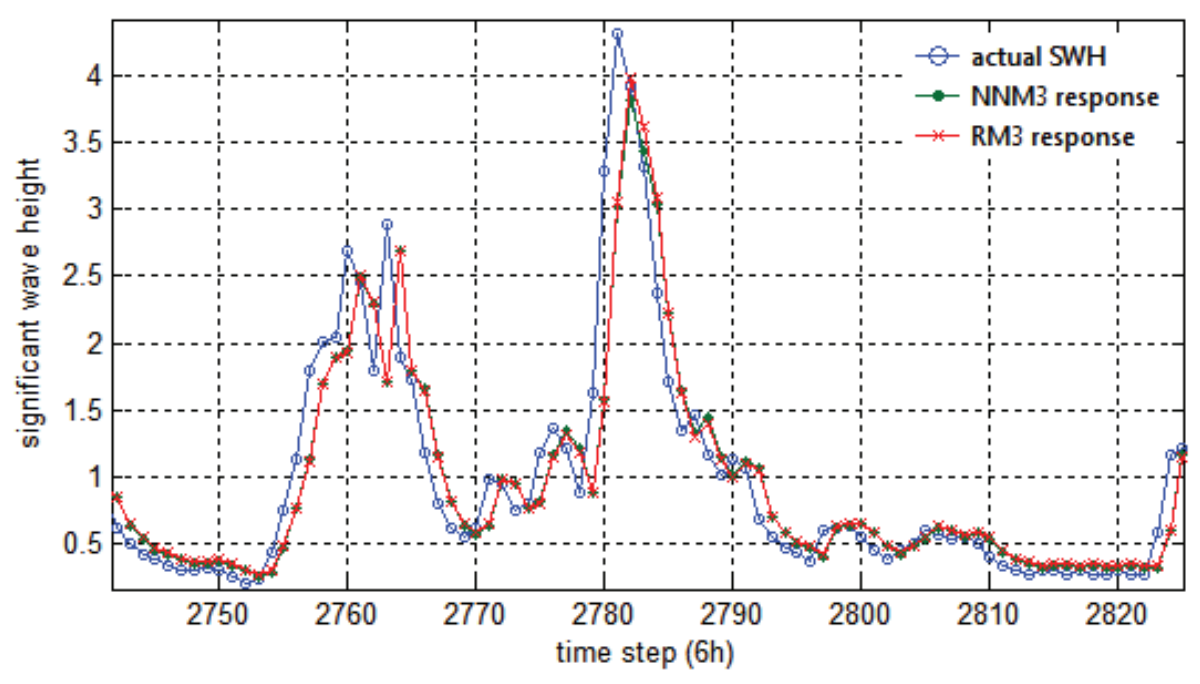

Figure 3.

Graphical comparison of model responses to actual SWH values from experiment 3.

In experiment 3, i.e. the case of $\mathrm{SWH}_{\mathrm{t}-1}$ used as a single input variable, the issue of models' responses lagging behind the actual SWH can be noted. This lag effect suggests the inability of the models to account for sudden changes of the output variable at the right time. The use of an adequate input variable, such as WSP, would announce the change and correct the error. Therefore, the best results are obtained when WSP ${ }_{t}$ and $\mathrm{SWH}_{\mathrm{t}-1}$ are used as inputs, as in experiment 2. In that case, $\mathrm{NNM}_{2}$ produces slightly better results than $\mathrm{RM}_{2}$, as can be seen from the numerical evaluation of the models.
Since the models performed equally in case of single input, and NNM performed better when two variables were used, neural network can be concluded to have certain advantages over regression in modelling complex processes where more input variables are expected to influence the output variable. Another advantage of a neural network is the simplicity of model formulation, which facilitates experimentation. As already noted, regression analysis requires the development of the optimum mathematical form, which is both time consuming and prone to errors. However, when neural network is used as a modelling 
tool, the network learns the optimum function by itself, without any assistance from the model designer.

The work presented in this paper is the first step in the modelling and prediction of the state of the Adriatic Sea based on available data. Further research should include modelling of other sea state variables for different points in the Adriatic Sea and prediction ability investigation, as well as prediction horizon determination. The model to be obtained by further research should provide reliable information for ship response modelling purposes, with the final goal of route optimization in heavy seas. That model should help build an efficient, simple, real-time decision making tool that could be used for navigation in bad weather in the Adriatic Sea, based on easily measurable data, i.e. wind speed and direction.

\section{ACKNOWLEDGMENTS}

This paper was supported in part by the Croatian Science Foundation under the 8658-DATAS project at the Faculty of Mechanical Engineering and Naval Architecture, Zagreb University. Company Fugro OCEANOR granted the academic license for the use of sea state data for the Adriatic Sea under the DATAS project. Authors Katalinić Marko and Mudronja Luka are PhD students at the Faculty of Mechanical Engineering and Naval Architecture and participate in the DATAS project.

\section{REFERENCES}

Beale, M. H., Hagan, M. T. and Demuth, H. B., (2010), Neural Network Toolbox 7 User's Guide, MathWorks.

Cybenko, G., (1989), Approximation by Superpositions of a Sigmoidal Function, Mathematics of Control, Signals and Systems, 2(4), pp. 303-314., https://doi.org/10.1007/BF02551274

de Vos, N. J. and Rientjes, T. H. M., (2005), Constraints of Artificial Neural Networks for Rainfall-runoff Modelling: Trade-offs in Hydrological State Representation and Model Evaluation, Hydrology and Earth System Sciences, 9, pp. 111-126., https://doi.org/10.5194/hess-9-111-2005

Foresee, F. D. and Hagan, M. T., (1997), Gauss-Newton Approximation to Bayesian Learning, Proc. of International Conference on Neural Networks, Houston, USA, June 9 - 12, vol. 3, pp. 1930-1935.

https://doi.org/ 10.1109/ICNN.1997.614194

Gupta, H. V., Sorooshian, S. and Yapo, P. O., 1999. Status of automatic calibration for hydrologic models: Comparison with multilevel expert calibration. Journal of Hydrologic Engineering, 4(2), pp. 135-143.
Haddadpour, S., Etemad-Shahidi, A. and Kamranzad, B., (2014), Wave Energy Forecasting Using Artificial Neural Networks in the Caspian Sea, ICE-Maritime Engineering, 167(1), pp. 42-52.

https:/doi.org/10.1680/maen.13.00004

Hagan, M. T. and Menhaj, M. B., (1994), Training Feedforward Networks with the Marquardt Algorithm, IEEE Transactions on Neural Networks, 5(6), pp. 989-993., https://doi.org/10.1109/72.329697

Haykin, S., (2005), Neural Networks: A Comprehensive Foundation, 9th Indian reprint, Pearson Education Inc.

Katalinić, M., Ćorak, M. and Parunov, J., (2014), Analysis of Wave Heights and Wind Speeds in the Adriatic Sea, 3rd International Conference on Maritime Technology and Engineering, Lisabon, Portugal, July 5 - 7, pp. 1389-1394.

Matić, P., Golub Medvešek, I. and Perić, T., (2015), System Identification in Difficult Operating Conditions Using Artificial Neural Networks, Transactions on Maritime Science, 4(2), pp. 105-112.

https://doi.org/10.7225/toms.v04.n02.001

Moriasi, D. N., Arnold, J. G., Van Liew, M. W., Bingner, R. L., Harmel, R. D. and Veith, T. L., (2007), Model Evaluation Guidelines for Systematic Quantification of Accuracy in Watershed Simulations, Transactions of the ASABE, 50(3), pp. 885-900.,

https//doi.org/10.13031/2013.23153

Ortiz-Royero, J. C. and Mercado-Irizarry, A., (2008), An Intercomparison of SWAN and WAVEWATCH III Models with Data from NDBC-NOAA Buoys at Oceanic Scales, Coastal Engineering Journal, 50(1), pp. 47-73.,

https://doir.org/10.1142/S0578563408001739

Peres, D. J., luppa, C., Cavallaro, L., Cancelliere, A. and Foti, E., (2015), Significant Wave Height Record Extension by Neural Networks and Reanalysis Wind Data, Ocean Modelling, 94(10), pp. 128-140.

https://doi.org/10.1016/j.ocemod.2015.08.002

Rawlings, J. O., Pantula, S. G. and Dickey, D. A., (2001), Applied Regression Analysis: a Research Tool, Berlin: Springer Science \& Business Media., https://doi.org/10.1007/b98890

Ris R. C., Booji N. and Holthuijsen L. H., (1999), A Third-generation Wave Model for Coastal Regions, Part II-Verification, Journal of Geophysical Research, 104(C4), pp. 7667-7681.

https://doi.org/10.1029/1998JC900123

Tabain, T., (1997), Standard Wind Wave Spectrum for the Adriatic Sea Revisited, Brodogradnja, 45(4), pp. 303-313.

Yu, H. and Wilamowski, B. M., (2011), Levenberg-Marquardt Training, In: Industrial Electronics Handbook, vol. 5 - Intelligent Systems, 2nd Edition, pp. 1-15.

Zamani, A., Azimian, A., Heemink, A. and Solomatine, D., (2009), Wave Height Prediction at the Caspian Sea Using a Datadriven Model and Ensemble-based Data Assimilation Methods, Journal of Hydroinformatics, 11(2), pp. 154 - 164., https://doi.org/10.2166/hydro.2009.043 